\title{
STAG2 Gene
}

National Cancer Institute

\section{Source}

National Cancer Institute. STAG2 Gene. NCI Thesaurus. Code C101346.

This gene is involved in cell division. 